\section{Beste Kasuistik des Jahres - Thieme-Kasuistikpreis 2020 der Phlebologie}

Im vergangenen Jahr wurden 8 Kasuistiken für die Phlebologie eingereicht und im Laufe des Jahres 2020 oder im Schwerpunktheft „Der besondere Fall“ veröffentlicht. Alle Beiträge standen damit auch zur Auswahl für den Thieme-Kasuistikpreis 2020. Nun ist die Entscheidung gefallen der Gewinner des Thieme-Kasuistikpreises 2020 der Phlebologie steht fest: Die Kasuistik „Postthrombotisches Syndrom auf dem Boden einer chronischen iliofemoralen Thrombose: Therapieoptionen und -limitationen“ (Phlebologie 2020; 49(05): 272-278) erhielt von allen eingereichten Kasuistiken die beste Bewertung der Jury aus Mitgliedern des Editorial Boards der Zeitschrift.
Die Kasuistik wurde von einem Autorenteam um Erstautor PD Dr. med. Andreas Bayer aus Kiel verfasst. Dieser schreibt zu dem Fall: „Nachdem wir initial aufgrund der reduzierten Erfolgsaussichten mit der Indikation zu dem vorgestellten Eingriff zurückhaltend waren, haben wir uns nach intensiver multidisziplinärer Diskussion der Befunde und Erfolgsaussichten auf ausdrücklichen Wunsch des Patienten zu diesem Eingriff - leider mit dem dargestellten Verlauf - entschieden.“ Die Autoren hoffen „mit der Präsentation dieser Kasuistik und unserer kritischen Diskussion dazu beitragen zu können, dass die Behandlung dieser komplexen vaskulären Pathologie zukünftig weiter optimiert und dadurch die postoperativen Langzeitoffenheitsraten nach diesen Operationen flächendeckend verbessert werden können.“
Aufgrund der aktuellen Situation kann der Kasuistikpreis leider nicht persönlich übergeben werden, die Urkunde wird dem Gewinner per Post zugesandt. Außerdem freuen wir uns, dem Erstautor der Kasuistik einen 1-jährigen kostenfreien Zugang zur Wissensplattform der eRef zur Verfügung stellen zu können. Ein herzliches Dankeschön an alle, die im letzten Jahr eine Kasuistik eingereicht und „ihren“ Fall zur Diskussion gestellt haben, sowie an die Jury des Kasuistikpreises.

Verlag und Schriftleitung 